\title{
Research into map-analysis strategies: theory- and data-driven approaches
}

\author{
LENKA HAVELKOVÁ, MARTIN HANUS
}

Charles University, Faculty of Science, Department of Social Geography and Regional Development, Centre for Geographical and Environmental Education, Prague, Czechia; e-mail: martin.hanus@natur.cuni.cz, 1.havelkova@natur.cuni.cz

ABSTRACT This study focuses on the strategies used during an analysis of four types of thematic maps (line symbols, area-shading, choropleths, and diagram maps) on the basis of the use of eyetracking technology. Moreover, it discusses the use of data-driven (spatial and spatiotemporal) and theory-driven (spatiotemporal) methodological approaches to research on the topic and aims to verify their applicability experimentally. The results of the experiment assist in identifying the main merits and limits of these approaches and serve to supplement and expand the results of prior studies. It is apparent that the strategies selected by users, when analyzing maps, are highly individualized and are distinguished by the frequent flitting of the eyes between the individual parts of the task. Among other things, it was discovered that most attention was paid to the task instructions and the map, and that the most frequently utilized sequence of eye movement was: task, map, (scale / north arrow, legend), answers.

KEY WORDS map use - strategy - mapping method - eye tracking - methodological approach

HAVELKOVÁ, L., HANUS, M. (2019): Research into map-analysis strategies: theory- and data-driven approaches. Geografie, 124, 2, 187-216.

Received November 2018, accepted March 2019.

(C) Česká geografická společnost, z. s., 2019 


\section{Introduction}

As a consequence of the wide availability of geo-information systems and the large volume of data which can be processed in the form of maps (Gołębiowska 2015), there has been an increase in the use of maps, particularly thematic maps, for the depiction of spatial information. It is therefore justifiable that the user-oriented aspects of (thematic) maps have come to the forefront of interest of researchers and educators (Havelková, Hanus 2018). Researchers and educators in this field have to cope with the diversity of the maps and the mapping methods used, and with the high variability of the cognitive operations related to map work.

Although a considerable amount has been written about what knowledge is essential in map work (Gerber, Lidstone, Nason 1992), map-related pedagogy is poorly developed, fragmented (Wiegand 2006) and highly dependent on the personal teaching concept of each teacher (Hanus, Havelková 2019). Even though significant progress has been made in research into map work over the last 35 years (Riding, Boardman 1983; van Dijk et al. 1994; Postigo, Pozo 1998; Clark et al. 2008; Ooms et al. 2015; Hanus, Marada 2016), there is still a need for an investigation into the cognitive mechanisms which underlie map work, specifically the strategies used when working with maps. This kind of exploration will be of significance for the teaching and learning of map work (Gerber, Lidstone, Nason 1992), as different users may interpret and process the same information in different ways (Ooms et al. 2012). Understanding the diversity in human behaviour when dealing with maps is therefore critical. This understanding can help educators when modelling the "optimal" (the most effective) strategy/strategies and, furthermore, can help in the identification of pedagogical and cognitive bottlenecks in the process of working with different (thematic) maps.

Considering the aforementioned, the principal goal of this study is to discuss the different methodological approaches used to assess map-analysing task strategies based on variable stimuli (i.e., maps designed using different mapping methods). The study subsequently aims to verify the applicability of these approaches by the experiment.

This will be achieved using eye tracking technology. Unlike previous studies (e.g., Çöltekin, Fabrikant, Lacayo 2010; Ooms et al. 2012), the experiment undertaken does not attempt to look for any similarities/differences between the participants' strategies, but rather between the tasks (i.e., between the individual map analysis operations and also between maps of different mapping methods). 


\section{The background}

Based on the goals of the study, it is clear that the focus is laid on the process of solving thematic-map-analysis tasks. More specifically, on the strategies, i.e., the sequences, of this task solving. The study thus links the approaches to map work research, conclusions of previous studies and the use of eye-tracking technology.

\subsection{Map work}

Map work involves understanding map concepts and practising map skills. Map skills can be further divided into map use and map design. Reading, analysing and interpreting a map can be included in map use. There is widespread agreement with regard to the increasing cognitive complexity of the subcategories of map skills, while map reading is considered to be a lower-order (less cognitivelydemanding) map skill and map analysis, interpretation and drawing higher-order (Wiegand 2006; Hanus, Marada 2016; Hanus, Havelková 2019). The categorization of map use skills is consistent with the objective-based taxonomies of cartographic interaction primitives distinguishing two or three main primitives, i.e., identify (commensurate with map reading), compare (commensurate with map analysis) and interpret (Roth 2012). However, as the below definition of map analysis indicates, map use skills are generally broader in their definition than the corresponding cartographic interaction primitives.

The experiment in this study focuses on map analysis skills. In essence, they require well-developed map-reading skills and constitute a prerequisite for map interpretation. From a geographical point of view, the basic map analysis skills involves: identifying and comparing the spatial distribution of the phenomena; and identifying the spatial relationships between the phenomena, the processes and the regions. Map analysis skills also include map scale use, self-location on a map and navigation (Wiegand 2006; Hanus, Havelková 2019).

\subsection{Related study}

Previous empirical research has investigated the question of whether the level of students' work with thematic maps depends on the chosen mapping method and, if so, to what extent, how and why (Havelková, Hanus 2018). The influence of mapping method on students' level of map reading, analysis and interpretation was examined using a sample of 392 students aged 17-20 with two qualitative (an area shading and a line symbols) and two quantitative (a choropleth and a diagram) maps. The participants were given a test consisting of four statements 
for each mapping method, which was used to investigate each of the three types of map skill (i.e., 48 true/false tasks in total).

Statistically significant differences between students, types of map skill and map types were observed from the overall results. Most participants were able to read the thematic maps without any substantial difficulties, but the map analysis skill was less developed and the level of thematic map interpretation, in particular, was significantly lower (Havelková, Hanus 2018, p. 155). Identifying the cause of these differences requires more detailed empirical research. It might be expected that one of the reasons is the different task-solving strategies used with different map types or the different strategies applied to different types of map skill. These task-solving strategies/sequences are a suitable subject for research via eye-tracking experiment (Çöltekin, Fabrikant, Lacayo 2010).

\subsection{Eye-tracking technology}

Studies using eye-tracking technology to identify task-solving strategies must mainly differentiate between two basic movements, i.e., fixations and saccades. During fixations, the eyes are fixed to one point of the perceived picture and the information depicted in that point is acquired and processed. It is precisely this combination of cognitive processes, attention and visual behaviour which forms the basis for the eye-mind hypothesis (Just, Carpenter 1976), on which the use of eye-tracking technology for the objective assessment of user strategies is based. As attention shifts, the eyes also carry out short, rapid movements between the individual fixations, known as saccades.

Thanks to the option of differentiating between these two movements and identifying their characteristics (i.e., their location, duration, frequency and sequences), we can evaluate not only the behaviour, and therefore also the strategies, of users when processing a wide variety of information or when solving a wide variety of tasks, but also the influence of selected independent variables on them (e.g., Mason, Pluchino, Ariasi 2014; Kim et al. 2015; Gołębiowska, Opach, Rød 2017). The option of evaluating the aforementioned characteristics of fixation in relation to the individual parts of the stimulus or the individual elements of the resolved task referred to as areas of interest (AOIs), is also regularly used during the visualisation and analysis of eye-tracking data.

\section{Methodological approaches}

Just as there are various methodological approaches for the investigation of strategies, so there are for their assessment and comparison. In general, it is possible 
to differentiate between data- and theory-driven approaches when assessing and comparing strategies. The majority of eye-tracking studies focusing on task-solving strategies make use of data-driven (bottom-up) approaches, because they attempt to categorise and thereby characterise the different types of user strategies based on empirical data from a limited sample of participants (e.g., Fabrikant et al. 2008; Mason, Pluchino, Ariasi 2014; Doležalová, Popelka 2016a; Gołębiowska, Opach, Rød 2017; Hsu, Chang, Liu 2018). On the other hand, theory-driven (top-down) approaches describe an individual's task-solving process from the point of view of its similarity with/differences from an optimal (hypothetical) strategy stated before-hand on the basis of a specific theory or an expert opinion (e.g., Çöltekin, Fabrikant, Lacayo 2010; Kim et al. 2015). Moreover, different methodological approaches focus on different aspects of the participants' task-solving process, as it is possible to look at it solely from a spatial point of view or to consider its temporal component concurrently.

\subsection{A spatial data-driven approach}

One of the most important characteristics of a task-solving strategy is its efficiency. This can be assessed on the basis of the total time spent solving the task, however, the time spent on the individual task elements provides us with more information (Opach, Gołębiowska, Fabrikant 2014). The proportion of time spent on each task element can be determined from eye-tracking data based on the number of fixations on a given element (Ooms, De Maeyer, Fack 2014). Furthermore, the number of transitions between task elements can be analysed in order to identify those elements whose information needs to be integrated according to the user's task-solving process (Hsu, Chang, Liu 2018). Moreover, the transitions enable researchers to, at least partially, observe the order which task elements are fixated by the user and whether the user has to return to any of the elements.

One drawback to this approach is that the aforementioned indicators can be substantially influenced by the difficulty of the task, which leads to difficulties when comparing strategies across tasks. Therefore, it is advisable to relativise the given indicators (Çöltekin, Fabrikant, Lacayo 2010; Ooms et al. 2012; Gołębiowska 2015).

\subsection{A spatiotemporal data-driven approach}

The assignment of tasks to a typology based on cognitive processes used during task solving can be undertaken on the basis of an analysis of the spatiotemporal similarities of the user strategies. Such an analysis can also reveal qualitative 
changes or, more specifically, the enhancement of strategies (i.e., the learning effect) during testing. In eye-tracking studies, eye movement sequences are compared during task solving, a so-called scanpath comparison. The scanpaths can be compared using various methods which differ in which aspects of the scanpath they focus on (see Anderson et al. 2015 for an overview).

One of the most commonly used methods of scanpath analysis is String-EditDistance which is based on sequence alignment analysis. In this method, scanpaths are replaced with strings of characters representing objects, i.e., areas of interests (e.g., task elements), in the order in which the user fixates on them (Fabrikant et al. 2008; Çöltekin, Fabrikant, Lacayo 2010; Opach, Gołębiowska, Fabrikant 2014; Doležalová, Popelka 2016a; Gołębiowska, Opach, Rød 2017). The degree of similarity between these strings is then calculated using one of the existing algorithms.

One of the most commonly used algorithms is the Levenshtein distance (Levenshtein 1966), which is defined as the lowest number of deletions, insertions or substitutions in a string required to transform it into another string. If transposition of string elements is also enabled during transformation, then it is a Damerau-Levenshtein distance (Damerau 1964). The Needleman-Wunsch algorithm (Needleman, Wunsch 1970), which is based on the identification of the number of concordant elements between two strings, offers a different approach to designating the similarity of the strings. However, these algorithms have been primarily designed to ascertain the similarities between identically or similarly long strings, which the participant fixation strings generally are not (Doležalová, Popelka 2016b). In light of these considerations, it is necessary to understand the mathematics behind the various algorithms and to ensure that the software chosen for scanpath comparison correctly compares strings of differing lengths and then correctly assigns them to clusters (for detailed explanation see Doležalová, Popelka 2016b).

\subsection{A spatiotemporal theory-driven approach}

Users' empirically ascertained strategies can also be compared with hypothetical strategies (Gerber, Lidstone, Nason 1992; Çöltekin, Fabrikant, Lacayo 2010; Kim et al. 2015). Optimal strategies, i.e., the most effective strategies for the successful completion of the given tasks, are most frequently considered. When designating the optimal strategies, it is advisable to proceed from significant theories from the areas of educational or cognitive psychology, such as the theory of learning, the theory of mental representation, the theory of cognitive load and other theories which are relevant to the expert-novice paradigm and the problem-solving process in general (Gagné 1977; Anderson 1983; Svenson 1992; MacEachren 1995; Haider, Frensch 1996; Paas, Renkl, Sweller 2003). 
The phases of task solving are subsequently designated on the basis of these theories. For example, Svenson (1992) defined several phases in the decisionmaking process: (1) problem recognition, (2) gathering information relevant to solving the problem, (3) evaluation of obtained information, (4) selection of the best option and (5) decision making. Nevertheless, the individual problem solving phases do not necessarily have to be generally designated in this way, but can be designated specifically for the given type of task and not merely on the basis of a certain theory, but also based on cognitive walkthrough sessions where experts consider possible usage scenarios (Polson et al. 1992).

When comparing the optimal strategies or the optimal scanpaths with the empirically identified user strategies, the same methods as those used in the aforementioned data-driven approach can be applied. However, qualitative methods, such as a detailed study of the recordings using the GazeReplay method, may also prove suitable, provided that there is multiple coding of the sequences by several researchers (for the purpose of eliminating any subjective coding).

\section{The experiment}

\subsection{The design and materials}

Both quantitative and qualitative research methods were used in the presented experiment (see the experiment design in Figure 1). Given the aim of the article, it was thought best to use eye-tracking testing as the main data source. Semistructured interviews were used as an additional method for identifying strategies and even more for understanding the reasons behind the choices and characteristics of the individual strategies.

The previously used achievement test (see Havelková, Hanus 2018) has been modified for the purposes of eye-tracking testing. The modified version consists of three tasks targeting the map analysis skill for each of the four fictional thematic maps using different mapping methods, namely line symbols, area-shading, choropleth and diagram mapping. Each of the twelve tasks was presented as a separate stimulus together with three possible answers (one correct answer and two distractors), the map and its compositional elements (the title, the thematic and topographic legend, the scale and the north arrow; see Figure 2 for the stimuli layout). The first tasks for the presented maps focused on the extraction of spatial distribution of phenomena. The second tasks required the use of the map scale. The third tasks described the spatial distribution using the cardinal points, so they involved the use of a north arrow. All of the tasks have been set out in Table 1 . 


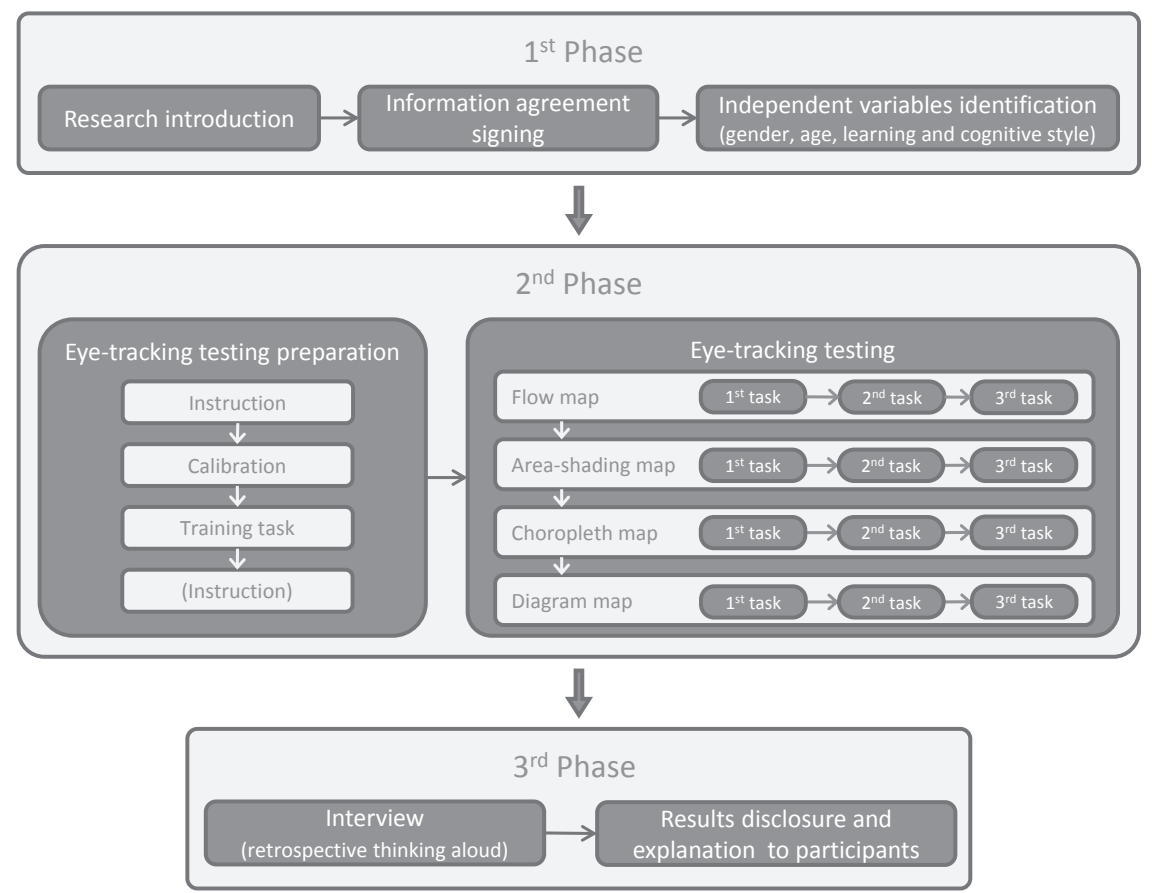

Fig. 1 - Experiment design

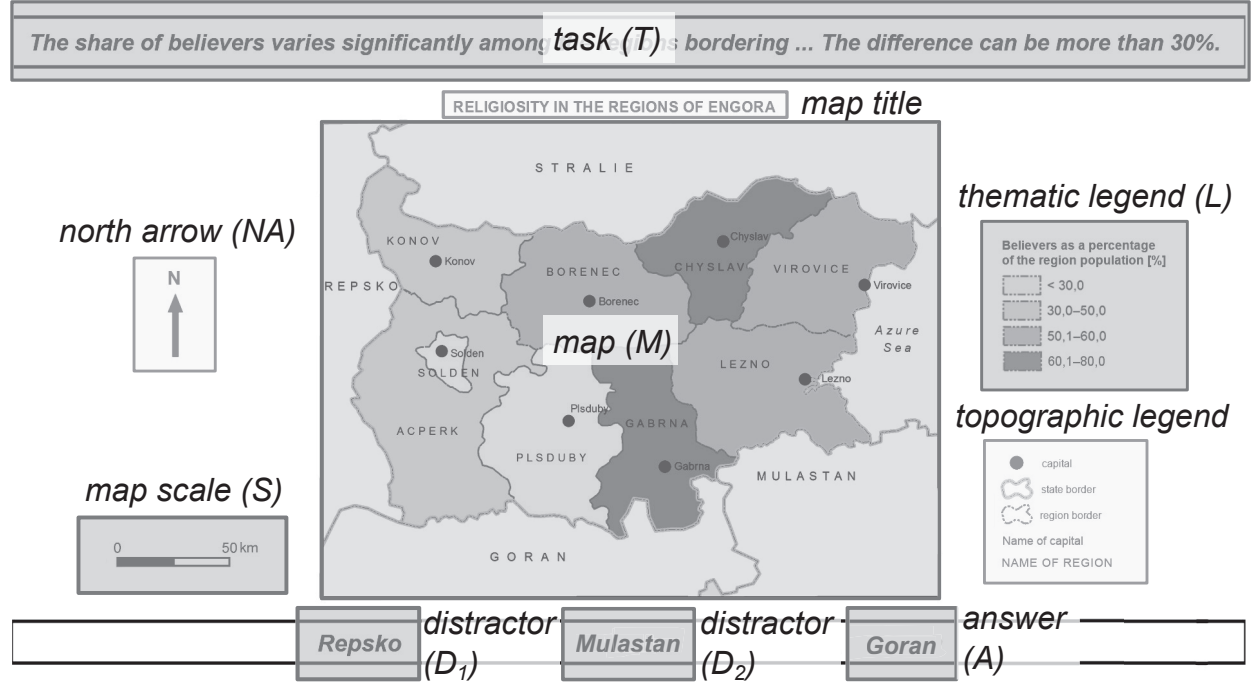

Fig. 2 - The stimuli layout with defined areas of interest. Note: The darker grey indicates areas of interests that were used in the data analysis and match with the areas of interest used in Figures 3,5 , and 6 . 


\subsection{The participants}

Nine geography majors ( 6 male and 3 female, aged 19 to 21 years) in their first year of university voluntarily participated in the study. All participants had normal or corrected-to-normal vision. Furthermore, they formed a relatively homogenous group from the point of view of the systematic development of map skills and did not routinely perform the types of tasks that were set during the eye-tracking testing.

\subsection{The procedure}

The participants were individually tested in a controlled laboratory environment at the Faculty of Science, Charles University, Prague, Czechia. Firstly, the general purpose and design of the study were explained to the participants and they were asked to sign an information agreement. The participants were subsequently given instructions regarding the eye-tracking testing along with a training task to verify their understanding.

Table 1 - The tasks used in the eye-tracking testing

\begin{tabular}{|c|c|c|}
\hline Mapping method & Task & Task code \\
\hline \multirow[t]{3}{*}{ Line symbols } & $\begin{array}{l}\text { All of the regional capitals neighbouring ... are connected by both a highway } \\
\text { and a railway. }\end{array}$ & $1 \mathrm{~A} 1$ \\
\hline & $\begin{array}{l}\text { Residents commuting by train from the capital of Chyslav to the capital of } \\
\text { Virovice travel approximately ... kilometres. }\end{array}$ & $1 \mathrm{~A} 2$ \\
\hline & $\begin{array}{l}\text { A highway running parallel to the ... coast of Engora connects Stralie and } \\
\text { Mulastan. }\end{array}$ & $1 \mathrm{~A} 3$ \\
\hline \multirow[t]{3}{*}{ Area-shading } & $\begin{array}{l}\text { We can find all the climate regions classified on the map in the regions } \\
\text { bordering... }\end{array}$ & $2 \mathrm{~A} 1$ \\
\hline & $\begin{array}{l}\text { An approximately ...-kilometre wide area of warm humid climate edges the } \\
\text { coast of the Azure Sea between the capitals of Lezno and Virovice. }\end{array}$ & $2 \mathrm{~A} 2$ \\
\hline & The warm dry climate area is located only in the .... of Engora. & $2 \mathrm{~A} 3$ \\
\hline \multirow[t]{3}{*}{$\begin{array}{l}\text { Choropleth } \\
\text { mapping }\end{array}$} & $\begin{array}{l}\text { The share of believers varies significantly between the regions bordering.... } \\
\text { The difference between the regions can even be more than } 30 \% \text {. }\end{array}$ & $3 \mathrm{~A} 1$ \\
\hline & $\begin{array}{l}\text { The distance between the regional capitals with the lowest share of } \\
\text { believers is less than ... kilometres. }\end{array}$ & $3 \mathrm{~A} 2$ \\
\hline & The share of believers in the ... of Engora is higher than in the ... of Engora. & $3 \mathrm{~A} 3$ \\
\hline \multirow[t]{3}{*}{ Diagram mapping } & Pines mostly grow in the forests in the ... regions. & $4 \mathrm{~A} 1$ \\
\hline & $\begin{array}{l}\text { The shortest distance from the Stralie borders to the region where only } \\
\text { coniferous forests are found is approximately .... kilometres. }\end{array}$ & $4 \mathrm{~A} 2$ \\
\hline & $\begin{array}{l}\text { Fir forests account for the fourth or lower largest share of the forests in all } \\
\text { the regions in the ... of Engora. }\end{array}$ & $4 \mathrm{~A} 3$ \\
\hline
\end{tabular}


The experiment had a within-subject design as all participants saw all twelve tasks (Table 1). The order of the mapping methods and tasks set in the previous study (Havelková, Hanus 2018) was preserved and, therefore, was identical for all participants (see Fig. 1). Due to the relatively small sample and the aim of identifying the influence of the learning effect, the option of rotating the tasks was not used.

The eye movement data were collected using the SMI RED $250 \mathrm{~Hz}$ system, which records at $250 \mathrm{~Hz}$ and was attached to a 15.6-inch monitor with a resolution of $1,920 \times 1,080$ pixels. The eye-tracking system was calibrated before each experimental session began. The fixation threshold was set at $80 \mathrm{~ms}$ (duration) and 50 pixels (dispersion radius) based on the general recommendations of Popelka (2014).

\subsection{Data analysis and visualisation}

The collected eye-tracking data were analysed using the OGAMA open source application (Voßkühler et al. 2008). Ten Areas of Interest (AOIs) were initially defined for all of the presented stimuli: namely task $(\mathrm{T})$, map $(\mathrm{M})$, correct answer $(A)$, first distractor $\left(D_{1}\right)$, second distractor $\left(D_{2}\right)$, map title, thematic legend $(L)$, topographic legend, map scale (S) and north arrow (NA) (see Fig. 2). However, the initial analysis showed that almost no fixations were recorded in the AOIs of the map title and topographic legend. The interviews subsequently confirmed that the participants hadn't used them, as they had found the information which they provided to be self-evident and therefore unnecessary when solving the task. As such, only the remaining eight AOIs were analysed during strategy identification.

In addition to the basic numerical characteristics of the eye-tracking data (i.e., task duration), the fixation count per AOI, the transition count between AOIs and the character strings representing the sequence of fixations with a character for AOIs hit were exported from OGAMA. In order to facilitate comparison of strings across participants, the resulting text files, each containing all strings from all participants for a given task, were then transposed into new text files, each containing all strings from all tasks for a given participant. Calculation of string similarity was carried out using the Scangraph online tool (www.eyetracking.upol.cz/scangraph) which offers a choice of three computational algorithms (namely the Levenshtein, Damerau-Levenshtein and Needleman-Wunsch) to compare sequences of different lengths (see Doležalová, Popelka 2016b for more information). 


\section{Results}

A total of 108 records (12 tasks for each of the 9 participants) were taken during the experiment. The analysis clearly shows that the range of implemented strategies differs not only among participants and map-analysing skills but also among the different thematic maps. The sequences differ both in the numbers of fixations (see Figure 3 for their distribution) and in their order. A total of 12,318 fixations in the defined AOIs were recorded, which is an average of 114 fixations per task and per participant. The shortest non-collapsed sequence comprised 31 fixations, while the longest comprised 357. In the case of collapsed sequences, the numbers were 11, and 79. The biggest average number of fixations was recorded in the tasks involving the ascertainment of spatial distribution in general (135 fixations per task per participant). In the case of tasks with cardinal points, the average was 108, while in the case of tasks involving the scale it was 99. Differences in the average number of fixations were also noted between individual maps: whereas the number was 166 fixations for line symbols, tasks with other maps were distinguished by tangibly lower values (area-shading: 110, diagram: 94 and choropleth: 87).

\subsection{The spatial data-driven approach}

Spatial data-driven analysis of participants' task-solving enables us to understand more deeply how and partially why the selected approaches to solving individual
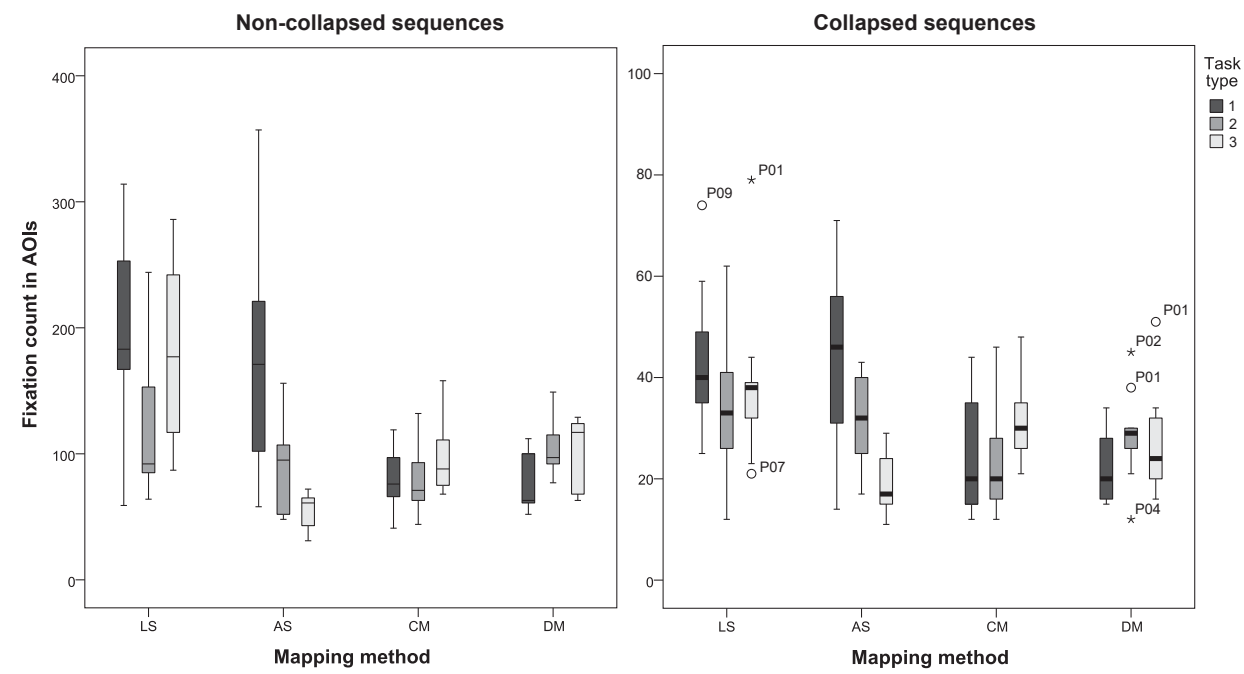

Fig. 3 - Distribution of fixation counts in AOIs for non-collapsed and collapsed sequences 


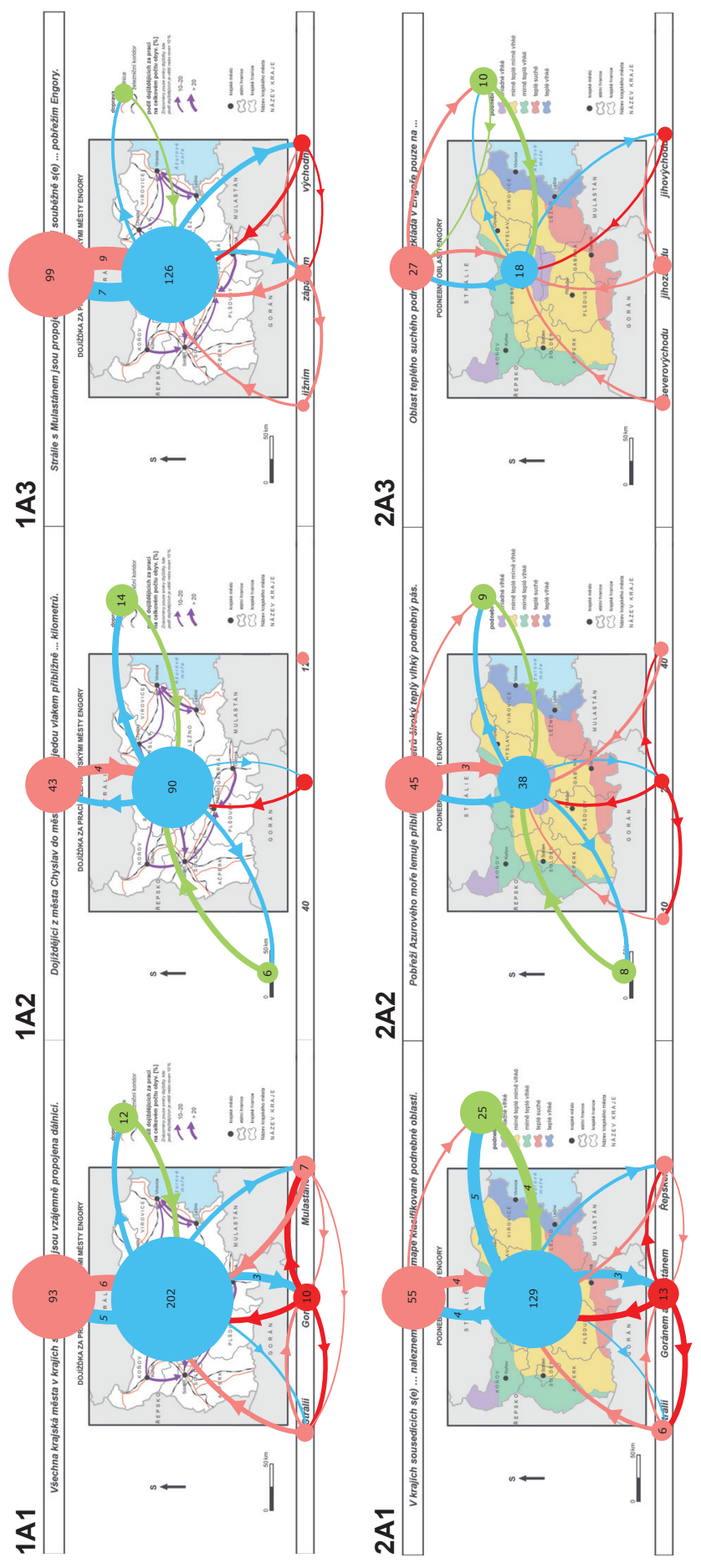



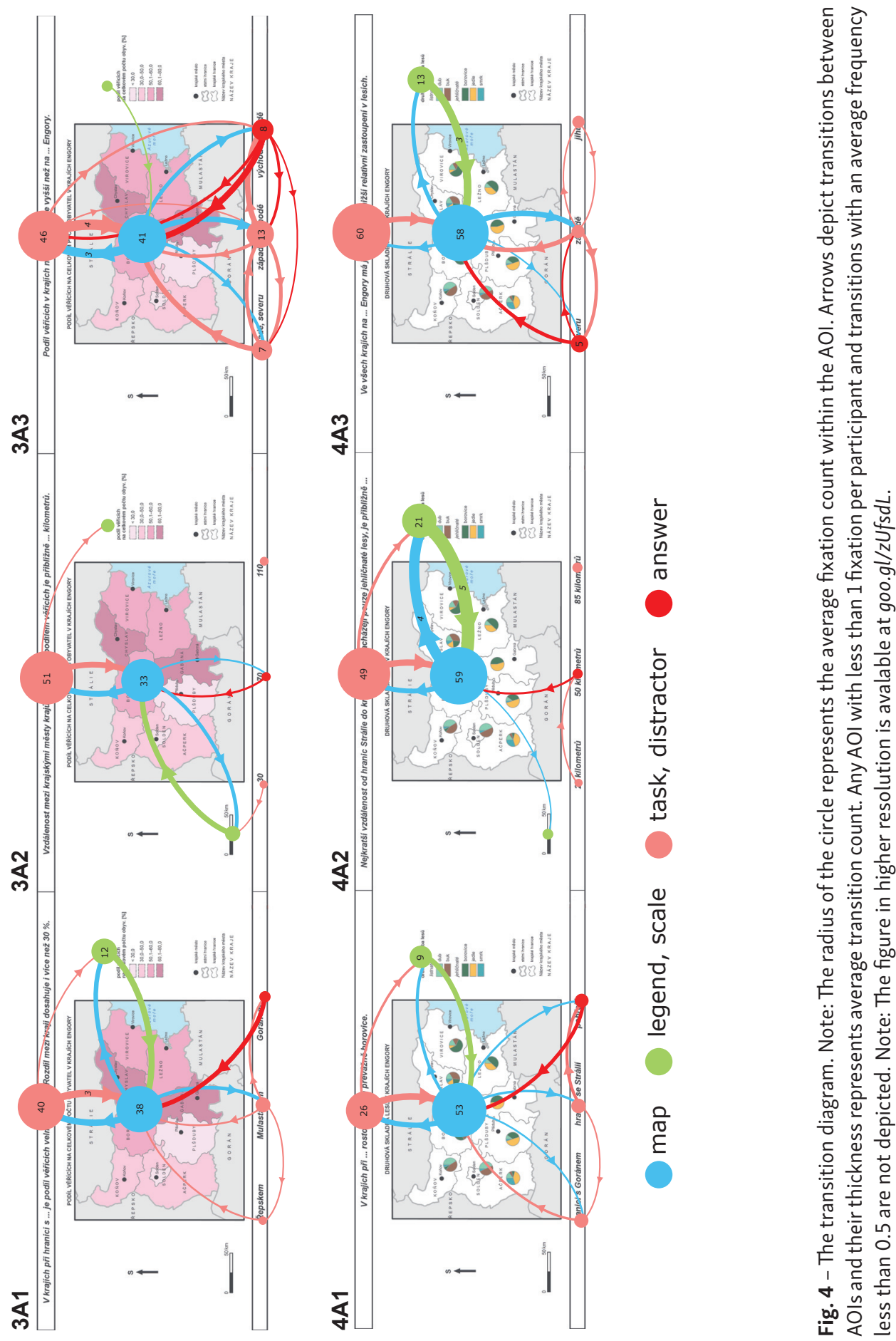
tasks are identical in some respects and different in others. The transition diagram visualising the fixation counts within AOIs and the transition counts between two AOIs averaged per participant (Fig. 4) has been created in order to simplify the identification of these similarities and differences. The separate transition diagrams for individual tasks are also available online as an additional file at goo.gl/zUfsdL. In addition to the transition diagram, a transition matrix (Fig. 5) has also been created in order to enable the comparison of the relativised fixation and transition counts.

An overall reduction in the average fixation count was identified over the course of the eye-tracking testing and this can be attributed to the reduction in the number of fixations in the map itself (see Fig. 4). The frequency of map fixating fell after the completion of the first task to an extent related to the given mapping method, which is highest in the case of line symbols and area-shading. The decrease in fixation counts in relation to mapping method is generally apparent throughout the course of the test, from earlier to later maps, with the partial exception of the diagram mapping. As such, the participants did not need as many fixations in the maps with stimuli which were presented later during the course of the experiment, despite the fact that the mapping method which was used to depict the data had changed.

Apart from the map itself, the second most important AOI is the task. These two AOIs jointly account for an average of $81 \%$ of the total number of fixations identified during task solving. They have the greatest relative importance in the case of task 1A3, where they account for $94 \%$ of all fixations (Table 1, Fig. 5). The number of fixations associated with the map and task AOI evened out as the fixation count within the map gradually fell (Fig. 4, Fig. 5). In some tasks (2A2, 2A3, 3A1, 3A2, $3 \mathrm{~A} 3$ and $4 \mathrm{~A} 3$ ), the participants even fixated more on the task than on the map on average, but the number of fixations on the task instructions remained relatively stable when subjected to an inter-task comparison.

Given the highly similar format and length of the instructions, the differing numbers of task fixations probably point to the tasks' differing levels of difficulty for the participants. In the case of the map legend, it was very clearly shown that the number of fixations within the AOI was associated both with what was required of the participant and with how easy it was for them to remember the legend or whether the symbology used was intuitive for them. As the transition diagram (Fig. 4) shows and as was subsequently confirmed by the interviews, the choropleth map's legend proved to be the most effective from this point of view.

As expected, a substantial number of fixations within the map scale AOI was only identified in those tasks which directly required the use of the scale (Fig. 4). The participants were, therefore, able to successfully recognise that they did not need this compositional element to solve the remaining tasks. However, no similar pattern emerged in the case of tasks which tested the ability to describe 
spatial distribution using the cardinal points. Most participants did not use the north arrow at all in these tasks (Fig. 4). As they stated in the interviews, they considered it to be a matter of course that the map should be oriented towards the north. Therefore, had the map orientation in the experiment been intentionally changed, the participants would probably had substantially lower success rates in this task type.

The spatial data-driven approach also enables identification of patterns in AOI transitions. It can generally be said that four transitions pairs stand out as most substantial, specifically between: the task and the map (an average of $28 \%$ of the transitions), the map and the legend (17\%), the map and the possible answers $(12 \%)$ and the individual possible answers (13\%; Fig. 5). Furthermore, the participants compared the individual possible answers with each other and with the information in the map less in the case of tasks requiring use of the map scale (Fig. 5).

Therefore, it can be generally stated that the first and the third tasks requiring use of the map scale for each map are similar from the point of view of transition patterns. The similar solution to these tasks was due to participants' intuitive perception of the orientation of the map when describing spatial distribution using cardinal points. However, despite this general trend, the aforementioned AOI transition patterns are not completely valid for all the tested tasks (e.g., 3A3 and $4 \mathrm{~A} 2)$.

\subsection{The spatiotemporal data-driven approach}

Despite the fact that the completion of some of the tasks could seem similar from the point of view of the spatial approach, this similarity does not continue once the temporal element of the task solution is taken into account. Similarly, patterns discovered at the general level do not have to apply at the level of individual participants and a more detailed analysis may point to important patterns at a lower level, which could be ascribed to the varied characteristics of the participants.

First, the Levenshtein, Damerau-Levensthein and Needleman-Wunsch algorithms and their various settings were compared (Table 2) in order to ascertain a setting which was able to find the similarities between sequences in sufficient resolution.

Given the different principles used to search for sequence similarities, both the Levenshtein and the Needleman-Wunsch algorithms were selected. The degree of similarity $(p)$ between collapsed strings in each algorithm was set differently in order to ensure that the number of discovered tasks which are mutually similar (i.e., edges in the graph) was similar (see Table 2). The differences between these algorithms in the tasks identified as being similar from the point of view of the collapsed sequences are clear from Figure 7. 


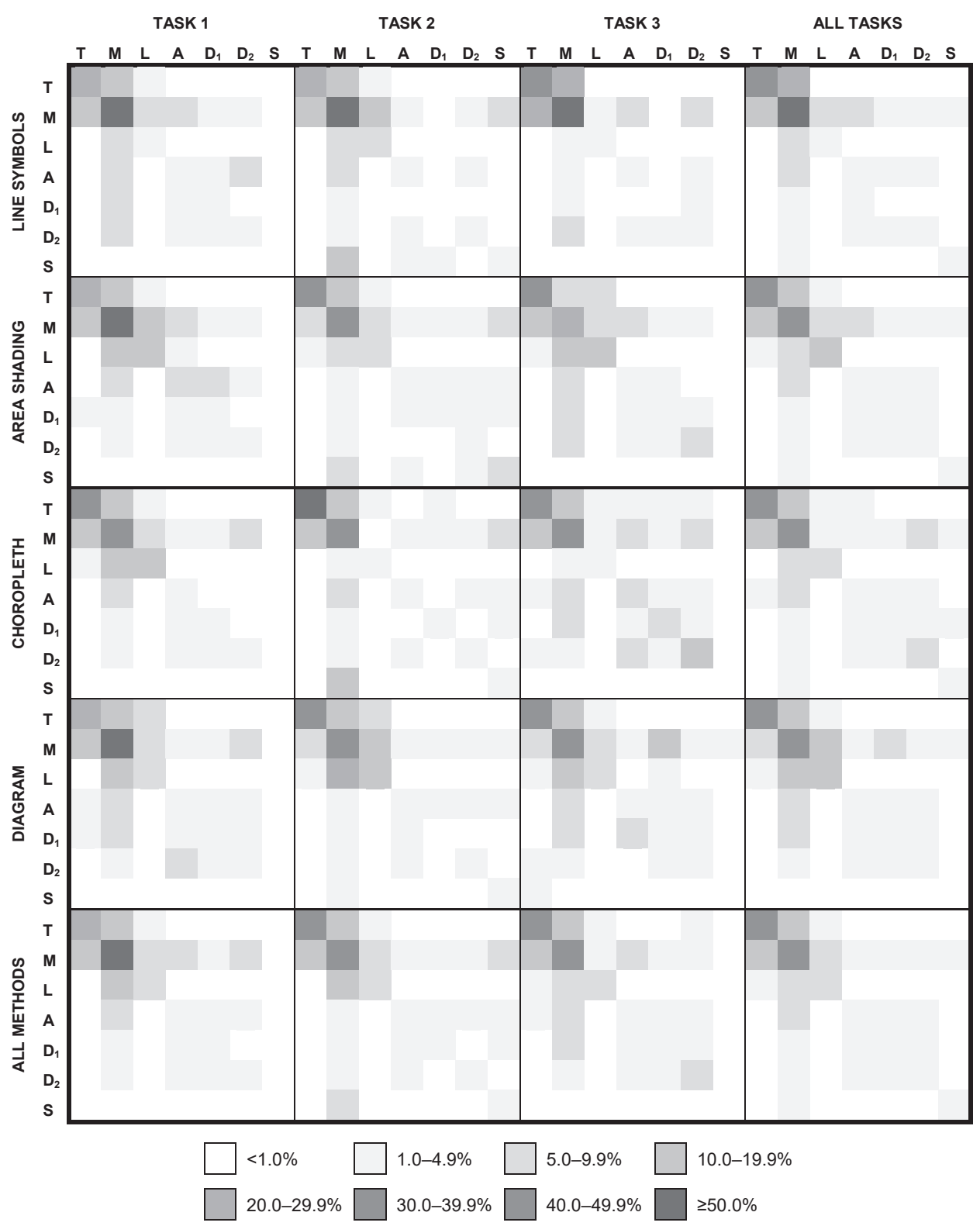

Fig. 5 - The relative transition matrix. Note: Colour of the diagonal cells corresponds to proportion of total AOI fixation count pertaining to given AOI. Other cells are colour coded based on percentage of total transition count between $\mathrm{AOI}$, which pertains to the transition between the two given AOIs. The north arrow AOI is not depicted in the matrix as the fixation and transition percentage was lower than $2 \%$ in every task. 
Table 2 - Number of detected edges in graph for individual tasks - a comparison of the algorithms implemented in Scangraph and their different calculation settings from the point of the string collapse and the degree of similarity $(p)$

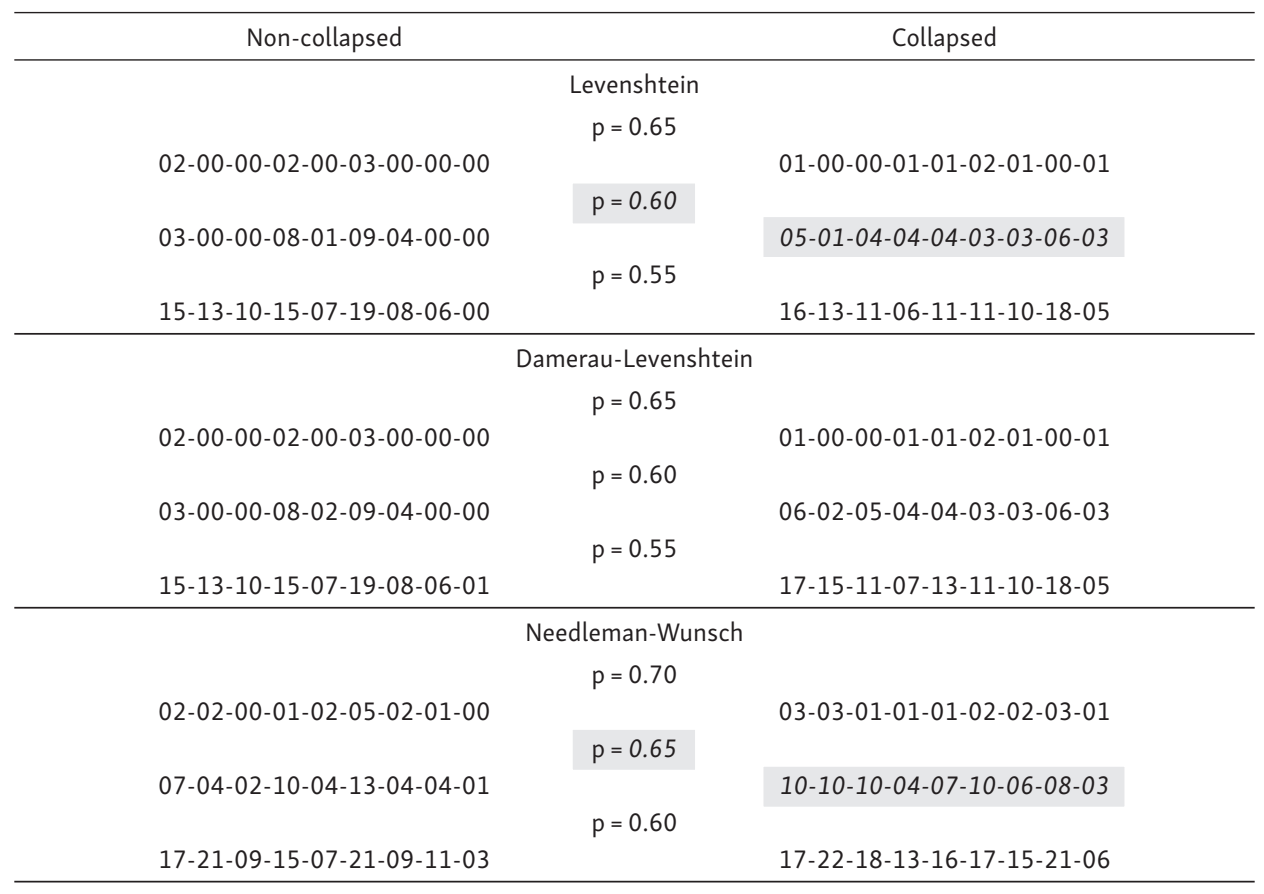

Note: The chosen settings are in italics.

The spatiotemporal data-driven approach confirmed that the participants had used similar task solving strategies, especially in the tasks focused on the description of spatial distribution phenomena (the $1^{\text {st }}$ tasks) and on the description of spatial distribution phenomena using the cardinal points (the $3^{\text {rd }}$ tasks; Fig. 6). However, the similarity of strategies used in tasks requiring the use of the map scale is not as conclusive. From this it is concluded that the participants were probably inconsistent as to in which task solution phase they used the map scale during the course of the eye-tracking testing. The differing importance of the legend when solving these tasks most probably also influenced it.

The increasing total number of edges identified for a given task and the more frequent agreement at the edges, i.e., in the ascertained similarity of the task solutions (Fig. 6), also points to modification of task-solving strategies during eye-tracking testing. Like Figure 4 from the spatial data-driven approach, Figure 6 similarly points to the fact that the choice of task solving strategies is independent of mapping method, i.e., that the map design does not critically influence the general form of the task solving method. 


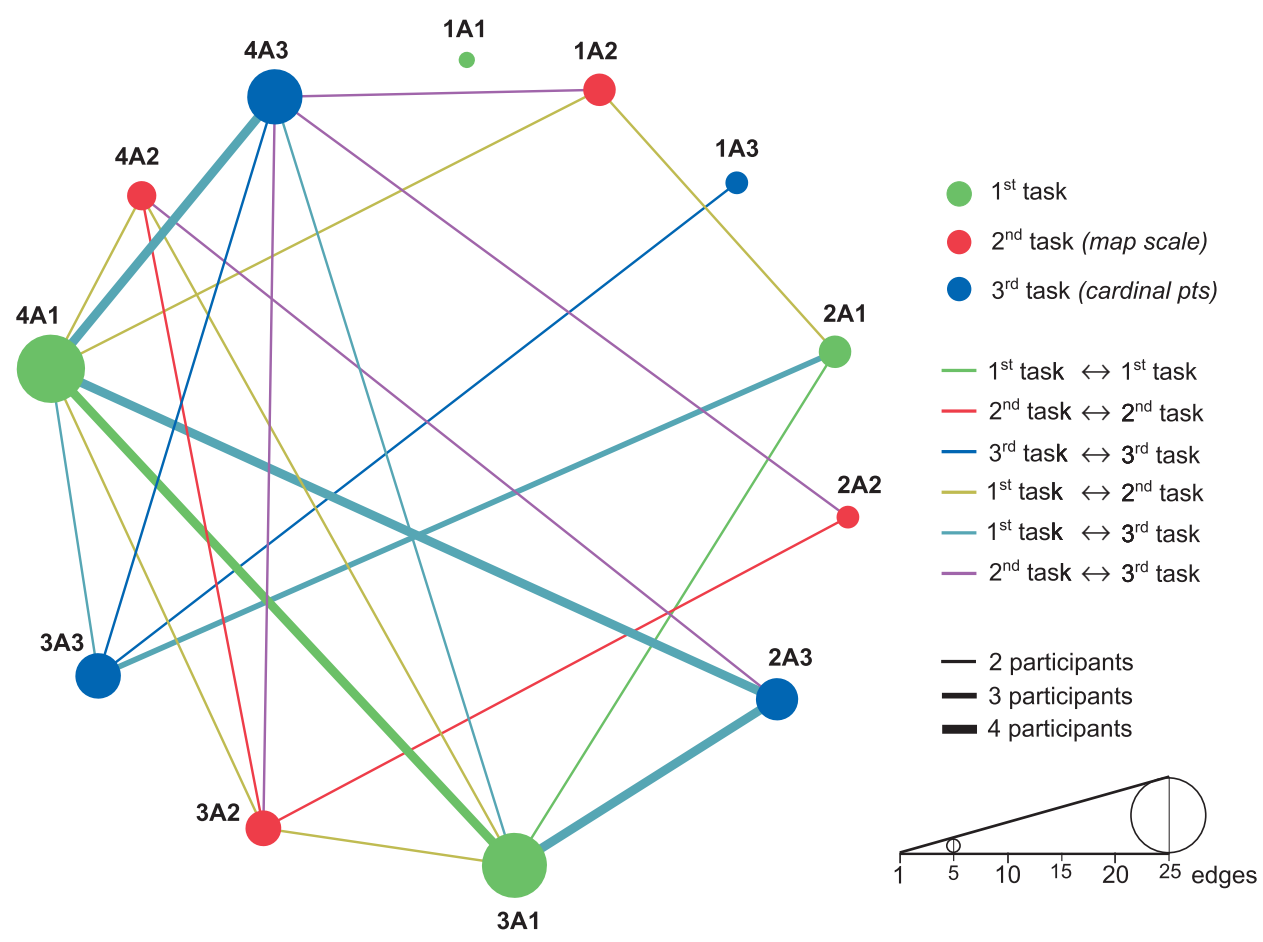

Fig. 6 - Summary of edges depicting the task similarity detected by at least one of the algorithms used. Note: Edge thickness represents number of participants for whom edge was identified. Edges which were only detected for one participant have not been included. Radius of circle presents total number of edges detected between given task and rest of the tasks.

The aforementioned patterns are observable for all participants when sequences are analysed altogether, but only for some participants when sequences are analysed by participant (compare Figure 6 with Figure 7). The influence of type of task on strategy is especially apparent in the case of participants 2, 4, and 9 if we disregard the intended differences between the first and third tasks. On the other hand, the results of the sequence analysis for the other participants indicate that the task-solving strategy is independent of both the given mapping method and the task type (Fig. 7).

Despite the fact that the degree of similarity was set at just $\mathrm{p}=0.65$ or $\mathrm{p}=0.60$, the number of similarly resolved tasks is low for some participants (Table 2, Fig. 7). However, all participants had a higher number of edges identified between the tasks which they solved during the second half of the eye-tracking testing, which means that the method of solving individual tasks gradually stabilised and, judging by the number of fixations and transitions, also became more effective (Fig. 4). Some participants also confirmed this gradually increasing 
Participant 1

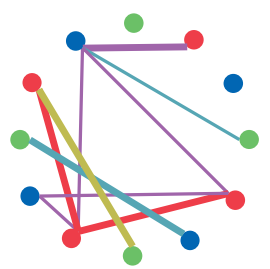

Participant 4

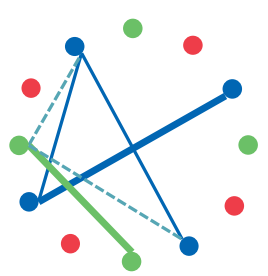

Participant 7

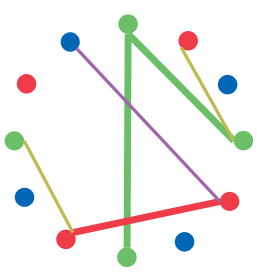

Participant 2

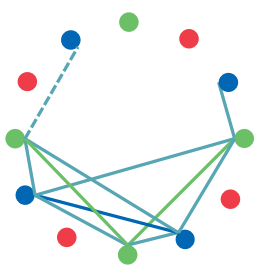

Participant 5

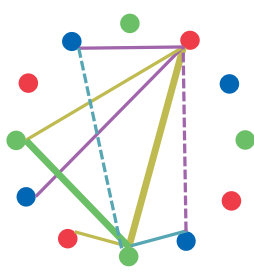

Participant 8

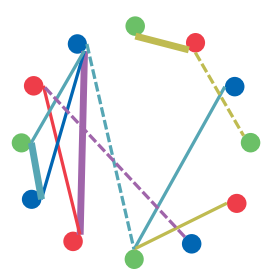

Participant 3

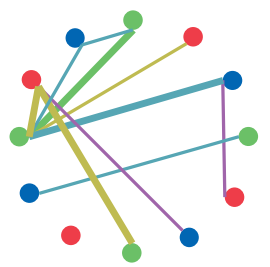

\begin{tabular}{ll}
- & Levenshtein \\
\hline & Needleman-Wunsch \\
both algorithms \\
& $1^{\text {st task }}$
\end{tabular}

Participant 6

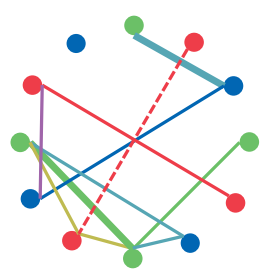

- $\quad 2^{\text {nd }}$ task (map scale)

- $\quad 3^{\text {rd }}$ task (cardinal pts)

$1^{\text {st }} \leftrightarrow 1^{\text {st }}$ task

$-2^{\text {nd }} \leftrightarrow 2^{\text {nd }}$ task

$-3^{\text {rd }} \leftrightarrow 3^{\text {rd }}$ task

$-1^{\text {st }} \leftrightarrow 2^{\text {nd }}$ task

Participant 9

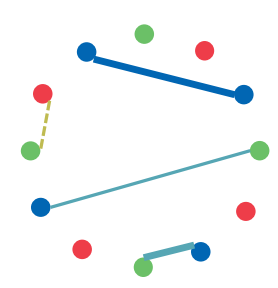

Fig. 7 - Edges depicting similarity of task solving used by individual participants based on Levenshtein $(p=0.60)$ and Needleman-Wunsch algorithms $(p=0.65)$

effectiveness and the discovery of suitable strategies in the post-experiment interviews.

\subsection{The spatiotemporal theory-driven approach}

Participant strategies were compared with the optimal, i.e., hypothetical, sequences in order to better understand how strategies were the same/different and strategy modification based on solved tasks. The hypothetical sequences were preset on the basis of cognitive walkthrough sessions where experts (cartographers and educators) considered possible usage scenarios (Polson et al. 1992).

The setting of hypothetical sequences for the task solving using the maps was based on Svenson's (1992) assumption that it is first necessary to become acquainted 


\begin{tabular}{|c|c|c|c|c|c|c|}
\hline $1 \sum$ & Task & Map & Answers & & & \multirow{2}{*}{ 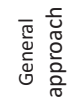 } \\
\hline 2 & Task & Answers & Map & & & \\
\hline 3 & Task & Legend & Map & Answers & & \multirow{4}{*}{ 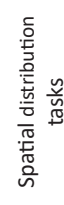 } \\
\hline 4 & Task & Map & Legend & Answers & & \\
\hline 5 & Task & Answers & Map & Legend & & \\
\hline 6 & Task & Answers & Legend & Map & & \\
\hline 7 & Task & $\sum$ Scale/North arrow & Legend & Map & Answers & \multirow{6}{*}{ 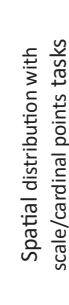 } \\
\hline 8 & Task & Legend & Scale/North arrow & Map & Answers & \\
\hline 9 & Task & Map & \rangle Scale/North arrow $\rangle$ & Legend & Answers & \\
\hline 10 & Task & $\sum$ Scale/North arrow $\rangle$ & Map & Legend & Answers & \\
\hline $11 \sum$ & Task & Answers & Map & Scale/North arrow & Legend & \\
\hline $12 \sum$ & Task & Answers & Scale/North arrow & Legend & Map & \\
\hline
\end{tabular}

Fig. 8 - Hypothetical sequences of AOls during task-solving process. Note: Capital letters in names of individual AOIs have been used in text and following figures as abbreviations in indicated strings. Colours designate type of sequence used in Figure 9.

with the problem or to study the task instructions. Two general scenarios (Fig. 8, sequences 1 and 2) were designated during the cognitive walkthrough with regard to the sequence of the subsequent steps.

At the same time, it is essential not only to work with the map as a whole but also to distinguish its compositional elements, such as thematic legend, scale, north arrow and the map itself, if we want to become acquainted with the strategy of map work. Various scenarios were drawn up with regard to the individual tasks during the cognitive walkthrough, taking into account the compositional elements which were considered to be essential for acquiring and assessing the relevant information (see Fig. 8).

The researchers subsequently looked into the use of these hypothetical sequences during task-solving by means of a detailed study of the GazeReplay recordings. This monitoring took place in two rounds. Basic strategy assessment parameters were designated in the first round. The highly frequent "flitting" between two parts of the task (AOIs; see also Figure 4) was monitored, and often made the resulting sequence opaque. In order to be able to compare a real sequence with hypotheticals, it was decided that any repeated transitions between two areas of interest would only be recorded once (e.g. TMTMTMTM becomes TM).

Svenson's (1992) approach was used during the assessment and it was stated that each new sequence starts with a study of the task. On the basis of this assumption, complex sequences were divided into partial sequences, which corresponded 


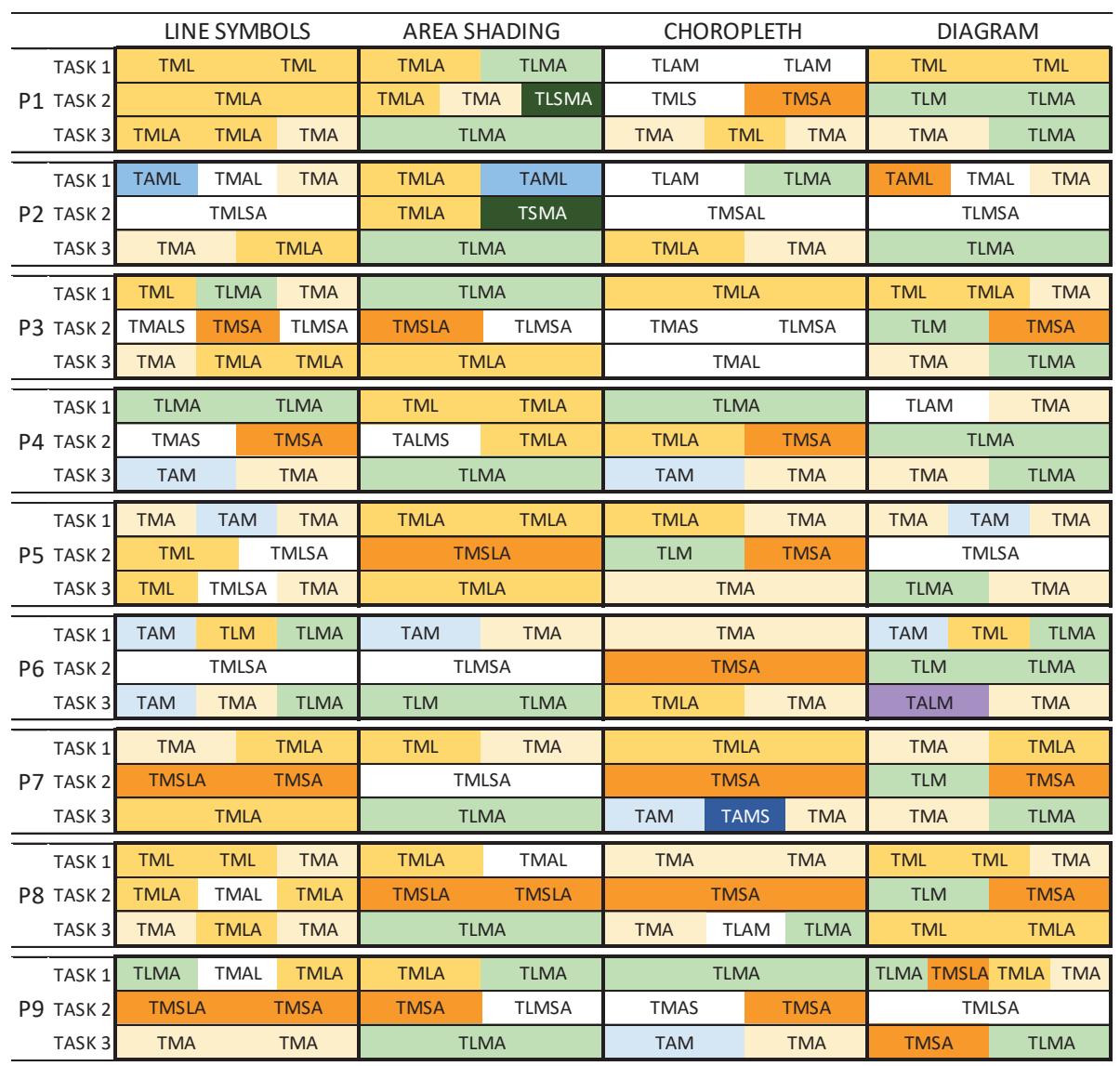

Fig. 9 - Hypothetical sequences used when solving individual tasks

relatively precisely to the pre-defined hypothetical sequences. The specific hypothetical sequences used during task solving were then identified in the second round of monitoring. Where sequences repeated in the record of a single task, the sequence was listed multiple times to distinguish between strategies comprising a single sequence and those comprising multiple repetitions of one sequence (see Figure 9). Svenson's (1992) approach was used to differentiate between these two types of strategy.

Participants solved some of tasks using a strategy which corresponded to one hypothetical sequence but, in other tasks, they used combinations of several hypothetical sequences (in some cases via a single, repeated sequence; in other via a combination of various sequences). The application of several sequences consecutively sometimes caused participants to omit a step (usually the last one) in the sequence, even though they were following a clear pattern. 
Table 3 - The most frequently used sequences (used in more than $10 \%$ of recordings) according to type of task and type of map

\begin{tabular}{|c|c|c|c|c|c|c|c|}
\hline \multirow{6}{*}{ 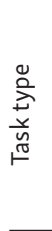 } & \multirow[t]{2}{*}{ spatial distribution (65) } & sequence no. & 4 & 1 & 3 & & \\
\hline & & $\%$ & 32.3 & 26.2 & 18.5 & & \\
\hline & \multirow[t]{2}{*}{ spatial distribution with scale (56) } & sequence no. & $\mathrm{N}$ & 9 & 8 & 4 & 3 \\
\hline & & $\%$ & 32.1 & 23.2 & 16.1 & 14.3 & 12.5 \\
\hline & \multirow[t]{2}{*}{ spatial distribution with cardinal points (69) } & sequence no. & 1 & 3 & 4 & 9 & \\
\hline & & $\%$ & 31.9 & 23.2 & 17.4 & 13.0 & \\
\hline \multirow{8}{*}{ 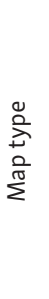 } & \multirow[t]{2}{*}{ flow map (53) } & sequence no. & 4 & 1 & N & 9 & \\
\hline & & $\%$ & 28.3 & 24.5 & 17.0 & 11.3 & \\
\hline & \multirow{2}{*}{ area-shading map (38) } & sequence no. & 4 & 3 & N & 9 & \\
\hline & & $\%$ & 31.6 & 26.3 & 13.2 & 10.5 & \\
\hline & \multirow[t]{2}{*}{ choropleth map (42) } & sequence no. & 1 & N & 4 & 8 & 3 \\
\hline & & $\%$ & 26.2 & 19.0 & 16.7 & 16.7 & 11.9 \\
\hline & \multirow[t]{2}{*}{ diagram map (57) } & sequence no. & 3 & 1 & 9 & 4 & \\
\hline & & $\%$ & 26.3 & 22.8 & 22.8 & 12.3 & \\
\hline
\end{tabular}

Note: Numbers of sequences correspond to numbers stated in Figure 8; $\mathrm{N}$ designates new sequences described in Figure 11. Number in brackets is total number of sequences used to solve given tasks.

It is clear from the results that the most frequently used hypothetical sequence (including use as a component sequence) is the approach: read task, study map, read answers, choose answer (sequence no. 1, TMA, used 40x). This sequence was also often used in a modified form, where map study was supplemented with legend study (as step three) (sequence no. 4, TMLA, used 41×). If we further enhance this sequence to include scale or the north arrow work (as step three between map study and legend study), we find another frequently used strategy (sequence no. 9, TMSLA, used 23×). The order of AOIs in these sequences shows that this involves the same map analysis strategy, which has simply been enhanced with other AOIs in some cases.

In addition to this strategy, the approach was also noted where instructions study was followed by legend study and only then by map study (sequence no. 3 , TLMA, used 35x) or the approach where, after studying the task's instructions, the participants also focused on the answers and only then dedicated their attention to the map (sequence no. 2, TAM, used 10x). Nevertheless, the sequence frequency differed both with regard to task type (and map analysis tested) and map type (mapping method; see Table 3 ).

It is obvious that strategies 1,3 and 4 were most common for all mapping methods, followed by strategy 9 for all map types except choropleth, where strategy 8 was more common. It can be inferred that this is due to the nature of this mapping method - after reading the task participants used the legend to verify values on the colour scale (without which it was not possible to read the relevant information from the choropleth maps). With other mapping methods, they studied the map 
Fig. 10 - Frequency of identified combinations when two or more sequences were used. Note: numbers of sequences on axes correspond to numbers stated in Figure 8; $\mathrm{N}$ designates new sequences described in Figure 11.

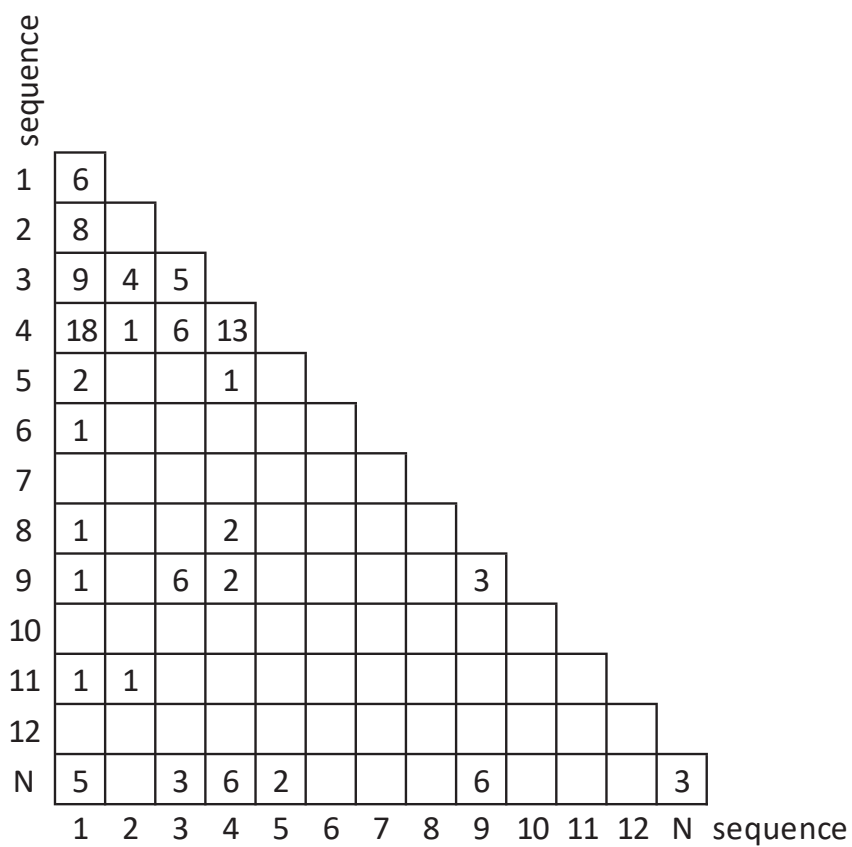

directly after reading the task and used the legend only if they came across an unknown or non-intuitive symbol.

Out of a total of 108 recordings, a single hypothetical sequence was used 33 times, while one sequence, whose approach was repeated, was used in a further 13 recordings. These recordings account for almost $45 \%$ of the sample. Those tasks with only one (possibly repeated) sequence most frequently used sequence 3 (TLMA: $15 \times$ ) and 4 (TMLA: 10x), followed by 9 (TMSLA: $6 \times$ ) and 1 (TMA: $4 \times$ ). The use of a single sequence (including repetitions) was recorded to a greater extent in the case of the work with the area-shading map (60\% of used sequences) and with the choropleth map (52\%), which differs substantially from the line symbol and diagram maps

Occurance

\begin{tabular}{|c|c|c|c|c|c|c|c|c|}
\hline$\sum$ & Task & $\sum$ & Legend & $\sum$ & Answers & Map & & $4 \times$ \\
\hline$\sum$ & Task & $\sum$ & Map & $\sum$ & Answers & Scale/North arrow & & $3 x$ \\
\hline$\sum$ & Task & $\sum$ & Map & $\sum$ & Answers & Legend & $>$ Scale/North arrow & $7 x$ \\
\hline$\sum$ & Task & $\sum$ & Map & $\sum$ & Legend & Scale/North arrow & Answers & $8 \times$ \\
\hline$\sum$ & Task & $\sum$ & Map & $\sum$ & le/North arrow & Answers & Legend & $1 \times$ \\
\hline$\sum$ & Task & \rangle & Legend & \rangle & Map & $>$ Scale/North arrow & Answers & $6 x$ \\
\hline
\end{tabular}

Fig. 11 - Newly identified task-solving sequences 
(both 30\%). The most frequent number of hypothetical sequences used in combination was two for the diagram map and three for the flow map (both 48\%).

The use of several sequences to solve tasks led to a relatively large number of different combinations. The most frequently used combination (Fig. 10) involved sequences 1 (TMA) and 4 (TMLA), followed by the two repetitions of sequence 4 (TMLA), combination of 1 (TMA) and 2 (TAM), and combination of 1 (TMA) and 3 (TLMA).

It was also ascertained that hypothetical sequence nos. 7, 10 and 12 were not used at all (not even as component sequences) and that sequences nos. 6 and 11 were only used in one case. Moreover, other sequences (used 29x) used during task solving (Fig. 11) had not been identified during the cognitive walkthrough.

\section{Discussion}

The results can only be considered preliminary from the point of view of strategies used when analysing thematic maps. This is especially the case with regard to the number of participants and partially with regard to the independent variables (i.e., task formulation), which could have unintentionally influenced the results. While keeping in mind the limitations, the results of the presented eye-tracking experiment have confirmed or discussed the conclusions of other studies involved in map work strategies or general problem-solving strategies.

\subsection{Discussion of results}

The results of this study have confirmed the opinions of experts who place map analysis among the more cognitively demanding map work skills (van Dijk et al. 1994; Wiegand 2006; Hanus, Marada 2016). As the first methodological approach showed and the theory-driven approach then confirmed, participants very frequently returned to individual task elements when solving tasks; for example, they had to read the instructions several times in a row or verify the meaning of individual colours/symbols in the map legend. It is therefore clear that they were unable to memorise all of the information which was necessary to solve the task and, as their task resolution sequences showed (Fig. 9), they sometimes even had to solve the entire task from the beginning again. As such, their method of task solving can be evaluated as ineffective from this point of view corresponding with behaviour often ascribed to novice users (Gerber, Lidstone, Nason 1992; Gerace 2001; Dimopoulos, Asimakopoulos 2010; Ooms et al. 2012).

As Anderson's ACT* theory (1983) indicates and the results of empirical studies have proven (Ç̈lttekin, Fabrikant, Lacayo 2010; Hsu, Chang, Liu 2018), novices are 
also distinguished by their non-uniform method of solving tasks, because their solution is bottom-up rather than top-down. The use of bottom-up methods is indicated by the relatively low number of similarities identified among task-solving strategies by sequence similarity analysis (Fig. 7) and the diversity (from the point of view of hypothetical sequences) of strategies used, even when solving a single task (Fig. 9). When using a bottom-up task-solving method, users do not have a pre-set solution method which they can uniformly apply to tasks of a similar type (Anderson 1983; Gerber, Lidstone, Nason 1992).

On the other hand, it is apparent from the results of the eye-tracking experiment that participants' strategy efficiency increased during testing (Fig. 4) and that this did not involve a strategy change, but can, at least in the case of some participants, be specifically designated as strategy enhancement, i.e., the learning effect (also analogously to Kim et al. 2015).

Novice users' strategies are also often substantially influenced by the visual appearance and saliency of individual stimulus elements (Çöltekin, Fabrikant, Lacayo 2010; Fabrikant, Hespanha, Hegarty 2010; Kim et al. 2015; Hsu, Chang, Liu 2018). It was therefore anticipated that the mapping method selected would influence the solution method. Nevertheless, task-solving strategy proved to be independent of this variable from the point of view of the majority of aspects investigated. In fact, quantitative mapping methods such as choropleth and diagram mapping appear to be less difficult from the point of view of strategy efficiency, in contrast to the conclusions of prior research (Havelková, Hanus 2018).

Participants' chosen task-solving strategies were admittedly not influenced by the mapping method selected, nonetheless, they may have been influenced by the choice of composition of stimuli, which was the same for all the tasks. When becoming acquainted with/reading certain material, it is natural to proceed from the top to the bottom (Fabrikant et al. 2008; Hsieh, Wu 2015). From this point of view, it is not surprising that participants proceeded in this manner in the majority of map-analysis tasks (Fig. 4, Tab. 3).

\subsection{Use of methodological approaches}

The experiment showed that it is advisable to combine all three mentioned methodological approaches when evaluating eye-tracking data from the point of view of strategies used. Each of these approaches can help unveil different aspects of the task-solving strategy used, i.e., its versatility, efficiency and possibilities for modification (enhancement). At the same time, the combination of several different methodological approaches at least partially filters out the approaches' specific limitations, which have to be taken into account during the data analysis itself and then subsequently during the interpretation of the acquired results. 
As in the case of the other two approaches, the use of the spatial data-driven approach is made difficult by the limited availability of data on visual processing by regularly used eye-tracking software. At the same time, visual comparison can prove problematic given a higher number of tasks or participants, which is, however, desirable for higher reliability.

The experiment also showed that the spatial data-driven approach can be highly useful when evaluating the validity and reliability of testing tasks. It can point to substantially different levels of difficulty in instructions for a specific task or to the fact that the influence of a certain independent variable has not been filtered out (e.g., stimulus composition, understanding of terms used or type of task formulation).

The spatial data-driven approach has only been used in the presented experiment to generally evaluate the strategy characteristics for the entire sample of participants but, nonetheless, as in the case of the other approaches, it is also possible to analyse similarity/differences at an individual level or between certain groups of participants (Ooms et al. 2012; Ooms, De Maeyer, Fack 2014; Opach, Gołębiowska, Fabrikant 2014).

The quantitative comparison of strings of characters, so-called String-EditDistance methods, is regularly used in studies when choosing the spatiotemporal data-driven approach. Various algorithms calculating degree of similarity may, however, evaluate other strategies as being similar to one another (see Figure 7). It is therefore advisable to compare the results of the various algorithms or to justify the algorithm selection. Similarly, it is important to critically interpret setting of sequence similarity, because, for example, it is possible to evaluate all participants as being similar to one another when setting a low degree of similarity (Table 2).

Nevertheless, as this research shows, a high degree of similarity should not be expected between tasks with long character strings, i.e. those which are more time-demanding for participants (Table 2). At the same time, degree of similarity is closely linked to number of differentiated AOIs. Therefore, it is necessary to choose carefully and use the algorithm to look for a more general task-solving strategy. The strategy can then be described in more detail, for example on the basis of qualitative analysis of eye-tracking data or of the subsequent interviews or retrospective "thinking aloud" process.

Any results acquired using String-Edit-Distance methods must also be combined with other methods of visualisation and eye-tracking data analysis, e.g., the GazeReplay method. Characterisation of the discovered clusters of similar strategies is especially difficult in the case of tasks which are time demanding, because even the individual collapsed strings consist of several tens of characters.

When categorising and characterising the different strategies in the case study, it also became apparent that giving a meaning to the vast amount of eye-tracking data can be simplified by theoretically embedding the experiment, i.e., by using 
the spatiotemporal theory-driven approach. The String-Edit-Distance method can be used in both the data- and theory-driven approaches and the hypothetical sequences can thus be compared with participants' sequences using some of the algorithms. However, this approach has proved unsuitable due to the substantially different string lengths. Quantitative comparison is therefore not suitable, especially in cases where users frequently return to individual task elements or flit between two AOIs. These transitions obscure the similarities/differences between strategies used at a general level during quantitative analysis.

Qualitative comparison of participants' sequences with hypothetical sequences can, therefore, help reveal more general natural relations, but it is time-consuming and requires the participation of more than one researcher to ensure adequate reliability. Moreover, the aforementioned length and characteristics of cognitively more demanding tasks mean that it is necessary (at least in the case of such tasks) to stipulate a suitable recording analysis system (e.g., rules for partial simplification of the sequence or division of task solution into several phases to be compared separately with the hypothetical sequences) and the coding of the strategies ascertained.

\section{Conclusion}

Research into map skills has long been part of geographic, cartographic and psychological literature, but it is now acquiring new impulses with the use of new technologies and methodological approaches. The eye-tracking technology used in this research has increased the importance of research into cognitive aspects of map work, within which it is possible to include research into strategies. It is precisely the identification of optimal/effective strategies for working with individual map types and solving variously cognitively demanding tasks which has the potential to be of great benefit in the area of the map skill development of the wider public. Responding the current lack of studies focusing on the cognitive demands of map skills, this study has discussed and experimentally verified three different methodological approaches. It's results have helped to identify the main merits and limits of these approaches and to supplement/deepen the results of the previous study, from which this case study has followed on (Havelková, Hanus 2018). The study has succeeded in identifying preliminary results, which need to be verified in a larger group of participants and contextualised with other variables already available, such as success rate in solving tasks (to identify successful strategies), cognitive type and learning style of participants (to determine suitable strategies for individuals); or with variables acquired during the study, for example, from the retrospective thinking-aloud interview (identification of bottlenecks in the map work process). 


\section{References}

ANDERSON, J.R. (1983): The Architecture of Cognition. Harvard University Press, Cambridge. ANDERSON, N.C., ANDERSON, F., KINGSTONE, A., BISCHOF, W.F. (2015): A comparison of scanpath comparison methods. Behavior Research Methods, 47, 4, 1377-1392.

CLARK, D., REYNOLDS, S., LEMANOWSKI, V., STILES, T., YASAR, S., PROCTOR, S., LEWIS, E., STROMFORS, C., CORKINS, J. (2008): University Students' Conceptualization and Interpretation of Topographic Maps. International Journal of Science Education, 30, 3, 377-408.

ÇÖLTEKIN, A., FABRIKANT, S.I., LACAYO, M. (2010): Exploring the efficiency of users' visual analytics strategies based on sequence analysis of eye movement recordings. International Journal of Geographical Information Science, 24, 10, 1559-1575.

DAMERAU, F.J. (1964): A technique for computer detection and correction of spelling errors. Communications of the ACM, 7, 3, 171-176.

DIMOPOULOS, K., ASIMAKOPOULOS, A. (2010): Science on the Web: Secondary School Students' Navigation Patterns and Preferred Pages' Characteristics. Journal of Science Education and Technology, 19, 3, 246-265.

DOLEŽALOVÁ, J., POPELKA, S. (2016a): Evaluation of the User Strategy on $2 \mathrm{~d}$ and 3d City Maps Based on Novel Scanpath Comparison Method and Graph Visualization. In: Halounová, L., Li, S., Šafář, V., Tomková, M., Rapant, P., Brázdil, K., Shi, W., Anton, F., Liu, Y., Stein, A., Cheng, T., Pettit, C., Li, Q. Q., Sester, M., Mostafavi, M. A., Madden, M., Tong, X., Brovelli, M. A., HaeKyong, K., Kawashima, H., Coltekin, A. (eds.): XXIII ISPRS Congress, Commission II. Copernicus Gesellschaft Mbh, Gottingen, 637-640.

DOLEŽALOVÁ, J., POPELKA, S. (2016b): ScanGraph: A Novel Scanpath Comparison Method Using Visualisation of Graph Cliques. Journal of Eye Movement Research, 9, 4, 1-13.

FABRIKANT, S.I., HESPANHA, S.R., HEGARTY, M. (2010): Cognitively Inspired and Perceptually Salient Graphic Displays for Efficient Spatial Inference Making. Annals of the Association of American Geographers, 100, 1, 13-29.

FABRIKANT, S.I., REBICH-HESPANHA, S., ANDRIENKO, N., ANDRIENKO, G., MONTELLO, D.R. (2008): Novel Method to Measure Inference Affordance in Static Small-Multiple Map Displays Representing Dynamic Processes. The Cartographic Journal, 45, 3, 201-215.

GAGNÉ, R.M. (1977): The conditions of learning. Holt, Rinehart and Winston, New York.

GERACE, W.J. (2001): Problem Solving and Conceptual Understanding. In: Franklin, S., Marx, J., Cummings, K. (eds.): Proceedings of the 2001 Physics Education Research Conference. PERC Publishing, New York, 33-45.

GERBER, R., LIDSTONE, J., NASON, R. (1992): Modelling expertise in map reading: Beginnings. International Research in Geographical and Environmental Education, 1, 1, 31-43.

GOŁĘBIOWSKA, I. (2015): Legend Layouts for Thematic Maps: A Case Study Integrating Usability Metrics with the Thinking Aloud Method. Cartographic Journal, 52, 1, 28-40.

GOŁĘBIOWSKA, I., OPACH, T., RØD, J.K. (2017): For your eyes only? Evaluating a coordinated and multiple views tool with a map, a parallel coordinated plot and a table using an eyetracking approach. International Journal of Geographical Information Science, 31, 2, 237-252.

HAIDER, H., FRENSCH, P.A. (1996): The Role of Information Reduction in Skill Acquisition. Cognitive Psychology, 30, 3, 304-337.

HANUS, M., HAVELKOVÁ, L. (2019): Teachers' concepts of map skill development. Journal of Geography, 118, 3, 101-116. DOI: 10.1080/00221341.2018.1528294.

HANUS, M., MARADA, M. (2016): What does a map-skills-test tell us about Czech pupils? Geografie, 121, 2, 279-299. 
HAVELKOVÁ, L., HANUS, M. (2018): The Impact of the Map Type on the Level of Student Map Skills. Cartographica, 53, 3, 149-170.

HSIEH, T., WU, K. (2015): The Influence of Gender Difference on the Information-Seeking Behaviors for the Graphical Interface of Children's Digital Library. Universal Journal of Educational Research, 3, 3, 200-206.

HSU, T.C., CHANG, S.C., LIU, N.C. (2018): Peer Assessment of Webpage Design: Behavioral Sequential Analysis Based on Eye-Tracking Evidence. Educational Technology \& Society, 21, 2, 305-321.

JUST, M.A., CARPENTER, P.A. (1976): Eye fixations and cognitive processes. Cognitive Psychology, 8, 4, 441-480.

KIM, K., KIM, M., SHIN, J., RYU, J. (2015): Eye-Movement Analysis of Students' Active Examination Strategy and Its Transfer in Visuospatial Representations. Journal of Geography, 114, 4, 133-145.

LEVENSHTEIN, V.I. (1966): Binary codes capable of correcting deletions, insertions, and reversals. Soviet physics doklady, 10, 8, 707-710.

MACEACHREN, A.M. (1995): How Maps Work: Representation, Visualization, and Design. Guilford Press, New York.

MASON, L., PLUCHINO, P., ARIASI, N. (2014): Reading information about a scientific phenomenon on webpages varying for reliability: an eye-movement analysis. Educational Technology Research and Development, 62, 6, 663-685.

NEEDLEMAN, S.B., WUNSCH, C.D. (1970): A general method applicable to the search for similarities in the amino acid sequence of to proteins. Journal of molecular biology, 48, 3 , 443-453.

OOMS, K., DE MAEYER, P., DUPONT, L., VAN DER VEKEN, N., VAN DE WEGHE, N., VERPLAETSE, S. (2015): Education in cartography: what is the status of young people's map-reading skills? Cartography and Geographic Information Science, 43, 2, 134-153.

OOMS, K., DE MAEYER, P., FACK, V. (2014): Study of the attentive behavior of novice and expert map users using eye tracking. Cartography and Geographic Information Science, 41, $1,37-54$.

OOMS, K., DE MAEYER, P., FACK, V., VAN ASSCHE, E., WITLOX, F. (2012): Interpreting maps through the eyes of expert and novice users. International Journal of Geographical Information Science, 26, 10, 1773-1788.

OPACH, T., GOŁĘBIOWSKA, I., FABRIKANT, S.I. (2014): How Do People View Multi-Component Animated Maps? The Cartographic Journal, 51, 4, 330-342.

PAAS, F., RENKL, A., SWELLER, J. (2003): Cognitive Load Theory and Instructional Design: Recent Developments. Educational Psychologist, 38, 1, 1-4.

POLSON, P. G., LEWIS, C., RIEMAN, J., WHARTON, C. (1992): Cognitive walkthroughs: a method for theory-based evaluation of user interfaces. International Journal of Man-Machine Studies, 36, 5, 741-773.

POPELKA, S. (2014): Optimal eye fixation detection settings for cartographic purposes. In: $14^{\text {th }}$ SGEM GeoConference on informatics, geoinformatics and remote sensing.

POSTIGO, Y., POZO, J.I. (1998): The Learning of a Geographical Map by Experts and Novices. Educational Psychology, 18, 1, 65-80.

RIDING, R.J., BOARDMAN, D.J. (1983): The Relationship between Sex and Learning Style and Graphicacy in 14-year-old Children. Educational Review, 35, 1, 69-79.

ROTH, R.E. (2012): Cartographic Interaction Primitives: Framework and Synthesis. The Cartographic Journal, 49, 4, 376-395. 
SVENSON, O. (1992): Differentiation and consolidation theory of human decision making: A frame of reference for the study of pre- and post-decision processes. Acta Psychologica, 80, $1-3,143-168$.

VAN DIJK, H., VAN DER SCHEE, J., TRIMP, H., VAN DER ZIJPP, T. (1994): Map skills and geographical knowledge. International Research in Geographical and Environmental Education, 3, 1, 68-80.

VOßKÜHLER, A., NORDMEIER, V., KUCHINKE, L., JACOBS, A.M. (2008): OGAMA (Open Gaze and Mouse Analyzer): Open-source software designed to analyze eye and mouse movements in slideshow study designs. Behavior Research Methods, 40, 4, 1150-1162.

WIEGAND, P. (2006): Learning and Teaching with Maps. Routledge, New York.

\section{ACKNOWLEDGEMENTS}

Funding for this research was provided by the Charles University Grant Agency, Project 42217, "Students' Strategies for Solving Tasks with Maps" and by Charles University Research Centre program no. UNCE/HUM/024.

The content of this Special Issue of Geografie Journal has been prepared together with the International Cartographic Association, namely with the Commission on Cognitive Issues in Geographic Information Visualization, the Commission on Use, User and Usability Issues, and the Commission on Atlases. Selected authors were contacted during the ICA Commissions' joint workshop "Atlases, Cognition, Usability" held in Olomouc (Czechia) at Palacký University in April 2018. The content of the Special Issue reflects the current topics handled by the Commissions and we hope that the special issue increases awareness and knowledge of cognitive and usability issues with geographic information among the broader geographic community. Preliminary selection of manuscripts and pre-submission communication with authors was done by Petr Kubíček (Masaryk University, proposed incoming co-Chair of the Cognitive Issues in Geographic Information Visualization Commission) and Amy Griffin (RMIT University, current co-chair of the Cognitive Issues in Geographic Information Visualization Commission). We thank all of the authors who contributed their research to this special issue. 